\title{
Polyarteritis nodosa of the liver: a report of two cases
}

\author{
Russell E. Cowan \\ M.B., M.R.C.P. \\ G. E. Thomas \\ M.A., F.R.C.Path.
}

\author{
C. N. Mallinson \\ M.B., F.R.C.P.
}

\author{
A. D. ThOMSON \\ B.Sc., Ph.D., M.R.C.P.
}

\section{Gastrointestinal Unit and Department of Pathology, Greenwich District Hospital, London SE10}

\begin{abstract}
Summary
Two patients with liver disease due to polyarteritis nodosa are described. They presented in a similar manner, with a swinging fever, a polymorphonuclear leucocytosis and high alkaline phosphatase levels, but the natural history of the illness was different, with recovery in one and death in the other.
\end{abstract}

\section{Introduction}

In the collagen syndromes, clinical liver disease is unusual and, if present, it is often due to secondary amyloidosis or to cardiac failure (Sherlock, 1975). In polyarteritis nodosa (PAN) the hepatic vessels may be affected but this is usually a post-mortem finding, when hepatic involvement varies from $42 \%$ (Mowrey and Lundberg, 1954) to $71 \%$ (Harris, Lynch and O'Hare, 1939) of cases. PAN is unlikely, therefore, to be suspected when patients present with liver disease, indicated only by abnormal liver function tests.

Two patients are described with PAN affecting the hepatic arterioles. Both presented with a swinging fever and polymorphonuclear leucocytosis for which there was no obvious cause, but high alkaline phosphatase levels indicated hepatic or biliary disease, and both were diagnosed from the histological appearances of liver biopsy specimens taken at laparotomy. Despite these similarities the natural histories of the illness differed, with recovery in one without corticosteroid treatment and death from systemic PAN in the other.

\section{Case 1}

The patient was 81 years old when she was admitted to hospital in 1973 with a history of diarrhoea without blood, fat or pus in the stool, for 6 months, intermittent fevers for 6 weeks and anorexia for 3 weeks. She also complained of progressive weakness and tenderness of the muscles of her thighs and

Correspondence: Dr R. E. Cowan, Department of Gastroenterology, The London Hospital, Whitechapel, London E1 1BB. shoulders, causing difficulty in rising from low seats. Six years before, diverticular disease of the colon had been diagnosed and for several years she had taken thyroxine $0.2 \mathrm{mg}$ daily and choline theophyllinate $200 \mathrm{mg}$ t.d.s.

Clinical examination revealed a swinging pyrexia for which there was no obvious cause. She was tender in the right hypochondrium, but no abdominal masses were palpable, and sigmoidoscopy to $15 \mathrm{~cm}$ was normal. There was weakness of extension of the neck and weakness and wasting of the gluteal muscles.

Investigations showed: haemoglobin $12 \cdot 8 \mathrm{~g} / 100 \mathrm{ml}$, total white blood count $16,900 / \mathrm{mm}^{3}$, with $87 \%$ neutrophils, ESR (Westergren) $45 \mathrm{~mm}$ in the first hour and blood urea $42 \mathrm{mg} / 100 \mathrm{ml}$. Repeated blood cultures were normal as was a midstream urine examination. Chest X-ray showed only several calcified foci and some generalized emphysema. Initial liver function tests showed: total bilirubin $0.3 \mathrm{mg} / 100 \mathrm{ml}$, alkaline phosphatase (AP) 173 i.u. (normal $<120$ i.u.) and aspartate aminotransferase 14 i.u. (normal $<20$ i.u.). Alkaline phosphatase isoenzyme studies showed the abnormal enzyme to be entirely of liver origin. Total serum protein was $4 \cdot 6 \mathrm{~g} / 100 \mathrm{ml}$ (normal $6 \cdot 5-7 \cdot 5 \mathrm{~g} / 100 \mathrm{ml}$ ) with albumin $2.2 \mathrm{~g} / 100 \mathrm{ml}$ (normal $3.0-4.5 \mathrm{~g} / 100 \mathrm{ml}$ ). The prothrombin time and other coagulation tests were normal. Barium enema showed a short segment of severe diverticular disease in the sigmoid colon, with some narrowing of the lumen. Oral cholecystogram failed to opacify the gall-bladder, which might have been due to liver dysfunction or to poor absorption of the contrast medium due to diarrhoea.

The swinging fever persisted and the AP rose to 321 i.u., and a laparotomy was performed 1 week after admission. A little clear peritoneal fluid was found but the gall bladder and biliary tree were normal. The liver was congested and enlarged, and a wedge biopsy was taken. The diverticula of the sigmoid were not inflamed and all other viscera were normal. 


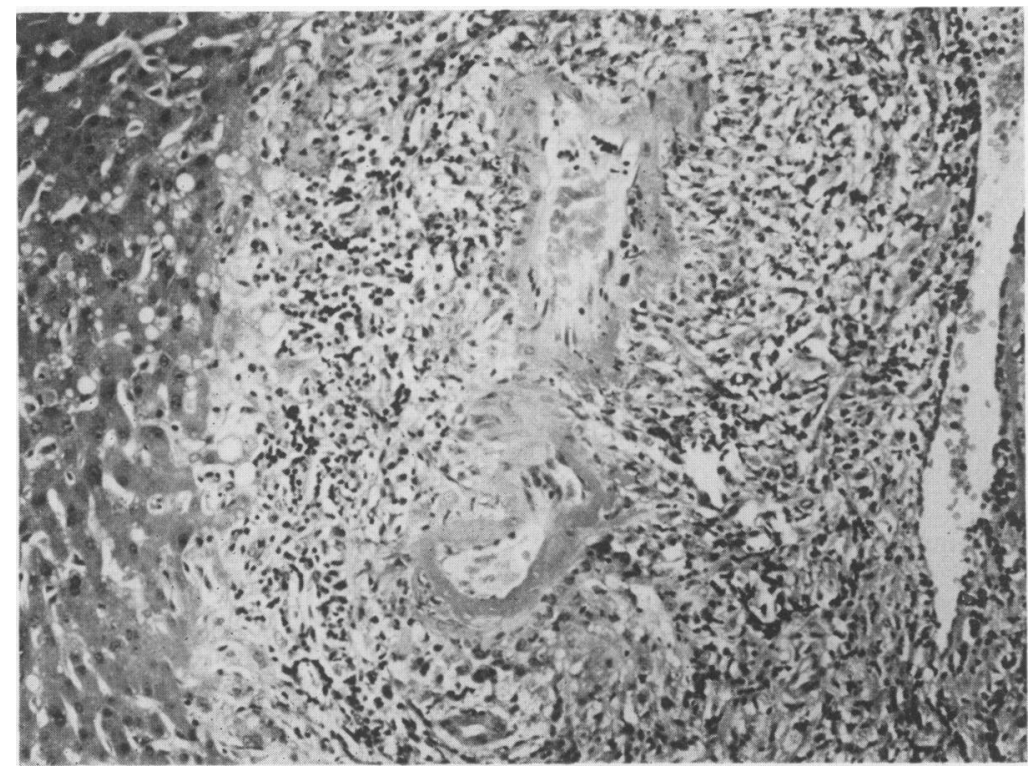

Fig. 1. Microscopic appearance of portal tract in liver biopsy specimen from patient 1 , showing fibrinoid necrosis of the media of two arterioles and heavy cellular infiltrate into the adventitia and perivascular tissues. $(\mathrm{H}$ and $\mathrm{E} \times 180$.)

Histological examination of the liver specimen showed fibrinoid necrosis of the media of two medium sized arterioles. The adventitia and perivascular tissues of these vessels were heavily infiltrated by neutrophils and moderate numbers of eosinophils, lymphocytes and plasma cells with a few histiocytes. It was concluded that these were changes of polyarteritis nodosa (Fig. 1).

After the operation the fever settled but the diarrhoea and muscle weakness persisted for several weeks and the anorexia worsened. Oedema of the arms and legs developed, the serum albumin having fallen to $1.9 \mathrm{~g} / 100 \mathrm{ml}$, while the blood urea rose to $116 \mathrm{mg} / 100 \mathrm{ml}$. She was treated with a high protein diet and diuretics and improved gradually with loss of oedema and diarrhoea, and return of muscular power and appetite.

Evidence of polyarteritis in other organs was sought. Serum creatinine was $1 \cdot 1 \mathrm{mg} / 100 \mathrm{ml}$. An intravenous pyelogram showed smooth outlines to the kidneys with no visible cortical narrowing. At no time was proteinuria detected. Electromyographic examination of the left deltoid and triceps muscles showed brief, polyphasic and low amplitude individual motor unit potentials which were consistent with a primary muscle disease. Muscle biopsy was not performed. Four weeks after surgery, selected angiography demonstrated a small liver with narrowing and uneven distribution of the arteries, especially the smaller branches, with some areas of under-filling. However, there was no definite angio -000 graphic evidence of polyarteritis in the arteries. $v$ examined or their branches. This included both renalo arteries. A $99 \mathrm{~m}$ Technetium colloid liver scan con-firmed a small liver but showed no other abnormality.

The patient was discharged after 3 months with only a little muscular weakness. At discharge AP was 164 i.u., total protein $5.9 \mathrm{~g} / 100 \mathrm{ml}$ and albumin $3.4 \mathrm{~g} / 100 \mathrm{ml}$. All other blood tests were normal.

Eighteen months after discharge she was symptomfree and all blood tests were normal. The liver scan was again normal as was an indocyanine green infusion test using $2.5 \mathrm{mg} / \mathrm{kg}$ body-weight. Hepatitis-B associated antigen and antibody were not found in the serum, and orcein staining of paraffin sections of the operative liver biopsy for the antigen in liver cells was negative. Tissue antibody studies revealed only a high titre of antibody to gastric parietal cells. A followup liver biopsy was thought to be unwarranted.

\section{Case 2}

The patient was 69 years old when admitted to hospital in 1954 with a 1 month history of swollen ankles and legs and aching in the calves and thighs. There was no significant past history.

On examination she was febrile but the swelling of the ankles and tenderness in the calves and thighs had cleared. The liver was felt five finger-breadths below the costal margin, but there were no other abnormalities. 


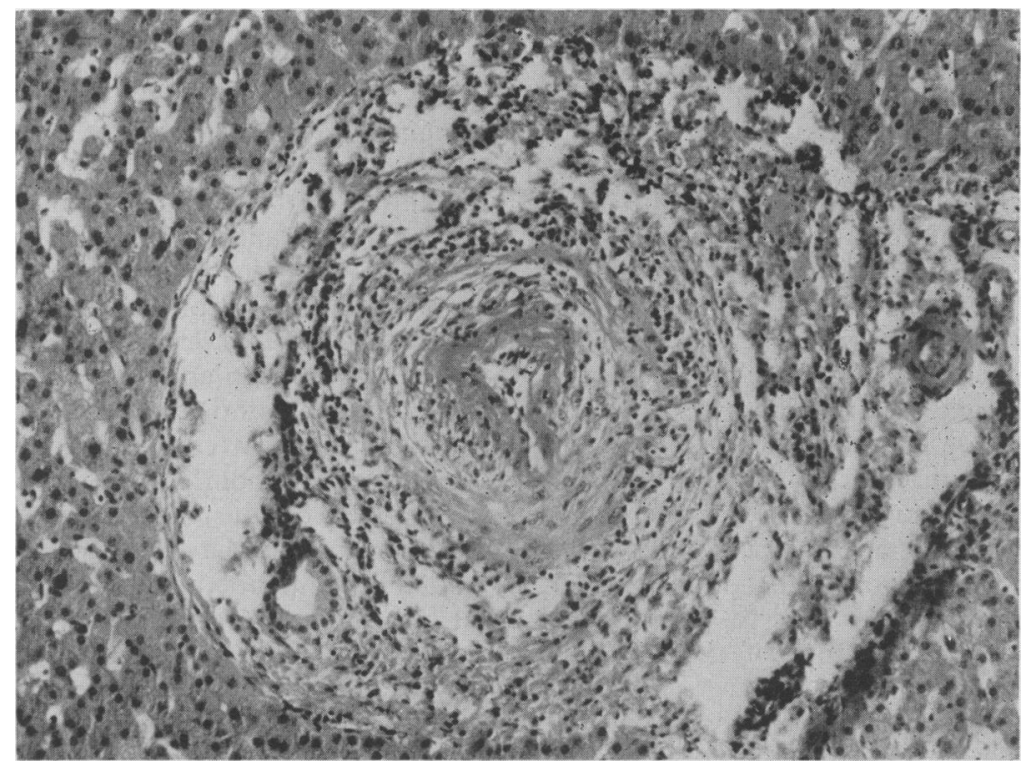

FIG. 2. Microscopic appearance of portal tract in liver biopsy specimen from patient 2 , showing fibrinoid necrosis of the media of one arteriole and cellular infiltrate into the adventitia. $(\mathrm{H}$ and $\mathrm{E} \times 180$.)

Investigations showed: haemoglobin $11 \cdot 2 \mathrm{~g} / 100 \mathrm{ml}$, mean corpuscular haemoglobin concentration (MCHC) $29 \%, \mathrm{MCH} 28 \%$ and total white blood count $22,500 / \mathrm{mm}^{3}$, with $93 \%$ neutrophils and $2 \%$ eosinophils. AP was $32.2 \mathrm{KA}$ units (normal $<16$ KA units), while other liver function tests, plasma proteins and blood urea were normal. A chest X-ray and the serum Wasserman reaction were also normal.

An Escherichia coli urinary infection was treated with sulphadiazine and the temperature fell. When a repeat urine culture was sterile 5 days later, the drug was stopped, but the white blood count remained at $22,000 / \mathrm{mm}^{3}$ and the fever recurred within $24 \mathrm{hr}$.

Further investigations, including blood cultures and agglutination tests for Salmonella and Brucella were negative. However, the bromsulphthalein retention test showed $16 \%$ retention of the dye at 45 $\min$ (normal $<5 \%$ ) and the AP remained raised, suggesting disease of the liver or biliary tree, supported by a non-functioning gall-bladder on oral cholecystography.

A laparotomy was performed which revealed a little straw-coloured peritoneal fluid and an enlarged liver from which a wedge biopsy was taken. The gallbladder and biliary tree were normal. The only other abnormality was a right ovarian tumour which was removed. This was firm and rubbery with a smooth and white surface; histological examination showed it to be a simple fibroma of the ovary. Many small arteries in its capsule and adjacent stroma showed fibrinoid necrosis of the media and an infiltration of histiocytes, eosinophils and many polymorphs into the adventitia, changes consistent with polyarteritis nodosa. The liver biopsy contained identical lesions in some of the portal tract arteries (Fig. 2).

She made an uneventful recovery from the operation and her symptoms slowly subsided. She was discharged symptomless 3 months after admission but with a persistent leucocytosis of $18,600 / \mathrm{mm}^{3}$, and a low grade pyrexia and hepatomegaly.

One week later she was re-admitted with a 4-day history of severe breathlessness and swelling of her legs. She was ill with signs of heart failure. Her liver was enlarged to six finger-breadths below the costal margin and was tender. She was now apyrexial.

An electrocardiogram suggested subendocardial myocardial infarction and treatment with digitalis and warfarin was commenced. The haemoglobin was $9.8 \mathrm{~g} / 100 \mathrm{ml}$ and $\mathrm{WBC} 19,400 / \mathrm{mm}^{3}$. She responded poorly to treatment and died 8 days after admission.

Post-mortem showed a haemorrhagic acute fibrinous pericarditis with $50 \mathrm{ml}$ of blood-stained pericardial fluid. There was no evidence of a myocardial infarction. The liver showed a number of scattered congested areas surrounding small, dense, white foci. Similar areas were seen on the surface of the spleen.

Lesions of polyarteritis nodosa were found in the liver, spleen, periadrenal fat, stomach, pancreas, tongue, uterus, cervix, kidney and left ovary. In 
addition there was considerable centrilobular congestion and necrosis in the liver, as well as patches of early calcification in the periportal fibrous tissue.

\section{Discussion}

The common clinical manifestations of PAN are well known. Mowrey and Lundberg (1954) found that a polymorphonuclear leucocytosis occurred in $72 \%$ of their 607 cases, and a variable fever, out of proportion to the clinical appearances, in $68 \%$. In contrast, clinical evidence of hepatic involvement is less common: Mowrey and Lundberg (1954) found that $21 \%$ of their cases had hepatomegaly but only $12 \%$ had jaundice and $6 \%$ abnormality of liver function tests. At post-mortem, however, they found liver involvement in $42 \%$ of 230 cases, while Harris et al. (1939) reported involvement of the hepatic arteries in $71 \%$ of eighty-seven cases, probably reflecting the widespread nature of the advanced disease.

The pathological changes of the blood vessels in the liver are similar to those in other organs. The hepatic arteries, arterioles and, occasionally, portal vein radicles, are involved by an inflammatory infiltrate of polymorphonuclear leucocytes, lymphocytes, eosinophils and plasma cells. Fibrinoid necrosis of the media may occur and fibrosis may follow with scarring and sometimes calcification of the periportal tissues. These histological appearances are probably specific to PAN (Mowrey and Lundberg, 1954). Intra- and extrahepatic arterial aneurysms, which sometimes rupture, may occur (Dzwonczyk, Serlin and Skerrett, 1959; Kanter, 1965). The main effect of these changes on liver parenchyma is hepatic infarction which was noted in $15 \%$ of 230 cases by Mowrey and Lundberg (1954). Pass (1935) collected fifty-four cases of hepatic infarction of which twenty-three were due to PAN.

Clinical presentation of PAN depends on the site and extent of the involvement. The two patients described here presented with liver disease, indicated only by raised serum levels of alkaline phosphatase, shown in the first case to be due entirely to the hepatic isoenzyme. In neither patient were the bilirubin or transaminase levels elevated. Although both had a swinging fever and a polymorphonuclear leucocytosis, their presentations otherwise were not specific for PAN. However, the disease in the second case followed the usual course, when untreated with corticosteroids, although there is little evidence to suggest that they improve long-term survival. The vasculitis had involved the arterioles of most organs at autopsy, causing death from congestive cardiac failure, secondary to haemorrhagic pericarditis, the result of vasculitis of the pericardial vessels. Conversely, the first case followed a benign course with complete recovery after 18 months, also without corticosteroids.

PAN is recognized as a complication of hepatitis B infection (Martini and Baltzer, 1972; Sestoft, Poulsen and Winkler, 1971), and a variety of other conditions. It is frequently associated with circulating surface antigen $\left(\mathrm{HB}_{\mathrm{s}} \mathrm{Ag}\right)$ or surface antibody (anti-HB s $_{\text {) }}$ to hepatitis B virus and with hepatitis B antigen-antibody complexes (Gocke et al., 1970; Trepo et al., 1974), and there is evidence to suggest that these antigen/antibody complexes may be related to the pathogenic mechanism (Trepo et al., 1974). In the first case $\mathrm{HB}_{\mathrm{s}} \mathrm{Ag}$ and anti-HB sere not present 18 months after discharge but they were not looked for during her illness, since when seroconversion may have occurred. However, orcein staining of the operative liver biopsy specimen was also negative, suggesting either the absence of $\mathrm{HB}_{\mathrm{s}} \mathrm{Ag}$ in the liver cells and the blood or an $\mathrm{HB}_{\mathrm{s}} \mathrm{Ag}$ associated acute hepatitis in which there is such extensive liver cell necrosis that orcein staining is absent (Deodhar, Tapp and Scheuer, 1975). Since there was no hepatitis in the biopsy specimen, the former explanation is more likely. These studies for hepatitis B infection were not available at the time of the second case.

Both patients demonstrate the diagnostic difficul ties of PAN when it presents as non-specific live disease without the features of one of its classicabe complications (Kanter, 1965; Fitchett, 1975). The diagnosis usually depends upon histological examination and in these patients was made from a wedge biopsy of the liver taken at laparotomy. Although this was not the indication for the operation, an operative liver biopsy may be essential with PAN of the liver to acquire a biopsy specimen large enough to make the diagnosis, since needle biopsy may be normal despite the presence of many hepatic vascular lesions (Bron and Gajaraj, 1970). These cases also indicate the rarity of this presentation of the disease, being the only examples at one hospital in 20 years.

\section{References}

Bron, K.M. \& GaJaraJ, A. (1970) Demonstration of hepatic aneurysms in polyarteritis nodosa by arteriography. New England Journal of Medicine, 282, 1024.

Deodhar, K.P., TAPP, E. \& Scheuer, P.J. (1975) Orcein staining of hepatitis $B$ antigen in paraffin sections of liver biopsies. Journal of Clinical Pathology, 28, 66.

Dzwonczyk, J., Serlin, O. \& SKerretT, P.V. (1959) Spontaneous rupture of the liver. Report of a case secondary to polyarteritis nodosa. Annals of Surgery, 150, 327.

FitchetT, D.H. (1975) Perihepatic haematoma in polyarteritis nodosa. Proceedings of the Royal Society of Medicine, 68, 805.

Gocke, D.J., Hsu, K., Morgan, C., Bombardieri, S., Lockshin, M. \& Christian, C.L. (1970) Association between polyarteritis and Australia antigen. Lancet, ii, 1149. 
Harris, A.W., Lynch, G.W. \& O'Hare, J.P. (1939) Periarteritis nodosa. Archives of Internal Medicine, 63, 1163.

KANTER, D.M. (1965) Hepatic infarction. Archives of Internal Medicine, 115, 479.

MARTini, G.A. \& Baltzer, G. (1972) Complications of viral hepatitis. Canadian Medical Association Journal, 106 (Suppl.), 508.

Mowrey, F.H. \& Lundberg, E.A. (1954) The clinical manifestations of essential polyangiitis (periarteritis nodosa) with emphasis on the hepatic manifestations. Annals of Internal Medicine, 40, 1145.
PASS, I.J. (1935) Infarction of the liver. American Journal of Pathology, 11, 503.

Sestoft, L., Poulsen, H. \& Winkler, K. (1971) Collagen disease debuting as acute hepatitis. Scandinavian Journal of Gastroenterology, 6, 495.

Sherlock, S. (1975) Diseases of the Liver and Biliary Tree, Fifth Edn, p. 531. Blackwell Scientific Publications, Oxford.

Trepo, C.G., Zuckerman, A.J., Bird, R.C. \& Prince, A.M. (1974) The role of circulating hepatitis $B$ antigen/ antibody immune complexes in the pathogenesis of vascular and hepatic manifestations in polyarteritis nodosa. Journal of Clinical Pathology, 27, 863.

\title{
Oxyphenbutazone-induced goitre
}

\author{
R. J. M. LANE \\ B.Sc., M.B., B.S., M.R.C.P. \\ F. Clark \\ M.B., B.S., F.R.C.P.

\section{J. K. McCollum* \\ F.R.C.S.}

\begin{abstract}
Departments of Medicine and *Surgery, Newcastle General Hospital, Newcastle upon Tyne
\end{abstract}

\begin{abstract}
Summary
A woman who had taken oxyphenbutazone for 4 years because of back pain presented with goitre and hypothyroidism. This was shown to be due to an organification defect, caused or aggravated by oxyphenbutazone.
\end{abstract}

\section{Introduction}

An adverse effect of some drugs lies in their goitrogenic action (de Groot and Stanbury, 1975). Phenylbutazone has this property and several reports of goitre and hypothyroidism associated with its administration have been published (Morgans and Trotter, 1955; Benedek, 1962; Kleint, 1971; RuizTorres, 1971; Vermulen and Schoot, 1972). It is not, however, widely appreciated that one of its major metabolites, oxyphenbutazone (Tanderil ${ }^{\$}$, Geigy) is also goitrogenic (Norrell, 1967; Miracco and Orlando, 1972). The following case of oxyphenbutazone-induced goitre is the first to be reported in Britain.

\section{Case history}

A 63-year-old woman was referred to surgical outpatients with a 2-month history of progressive thyroid

Correspondence: Dr R. J. M. Lane, c/o Renal Office, Newcastle General Hospital, Westgate Road, Newcastle upon Tyne NE4 6BE. enlargement and symptoms of hypothyroidism. On examination, she had a large, firm, vascular, nontender goitre and was clinically hypothyroid. There was no family history of thyroid disease and she ate a normal, balanced diet. She gave a long history of low back pain and 5 years previously she had taken phenylbutazone and dihydrocodeine for about 1 year. She had subsequently been found to have marked hyperuricaemia and had been put on allopurinol $100 \mathrm{mg}$ t.d.s. and oxyphenbutazone 100 mg t.d.s., which she had taken regularly for 4 years. Thyroid function tests confirmed hypothyroidism (see Table 1) and no anti-thyroid antibodies were detectable.

Assuming iodine deficiency to be unlikely, the results suggested that the hypothyroidism was due to an organification defect. This was confirmed by a perchlorate discharge test (see Fig. 1). Uptake of ${ }^{131}$ I was very high $(32 \%$ at $30 \mathrm{~min})$ but following the administration of potassium perchlorate at $40 \mathrm{~min}$ there was a discharge of isotope from the gland, so that at $70 \mathrm{~min}$, only $28 \%$ of the administered radioactivity remained in the thyroid.

All medication was withdrawn and over the next 4 weeks the patient became clinically and biochemically euthyroid (PBI $549 \mathrm{~nm} / \mathrm{l}$, TSH 4.6 nu./1). Twenty-one months later she remained euthyroid and the thyroid 